\title{
LA REPRESENTACIÓN SOCIAL DE FAMILIA EN LAS MUJERES INDÍGENAS MIGRANTES DE TABASCO
}

\author{
Dra. Crystiam del Carmen Estrada Sánchez ${ }^{1}$ \\ Dr. José Antonio Morales Notario ${ }^{2}$
}

Resumen: Esta investigación tiene como objetivo reflexionar sobre las representaciones sociales en un estudio de caso: La representación social de familia en las mujeres indígenas migrantes de Tabasco. El análisis se centra particularmente en las familias de Edilia López Martínez, Ceferina Rodríguez Martínez y Martina Fernández Pérez. Las dos primeras son familia, son primas, la última sólo es parte de la misma comunidad y se sustenta en la Teoría de las Representaciones Sociales, definida en Prácticas sociales y representaciones sociales de Jean Claude Abric (2001) "[...] como una visión funcional del mundo que permite al individuo o al grupo conferir sentido a sus conductas y entender la realidad mediante sus propios sistemas de referencias y adaptar y definir de este modo un lugar para sí".
De acuerdo a este pensamiento, las representaciones sociales poseen una estructura con elementos que la distinguen: el núcleo central y las periferias. La primera con una función generadora y organizadora, y las últimas con funciones de concreción, regulación y defensa. Con estos dos puntos y sus componentes, las mujeres indígenas explican lo que significa la representación familiar para ellas.

Palabras-Clave: Representación social. Familia, mujeres. Migrantes, indígena

\begin{abstract}
The aim of this research is to think about social representations in a case study. The social representation of families in indigenous immigrant women in Tabasco. The analysis focuses on the families of Edilia López Martínez, Ceferina Rodriguez Martinez and Martina Fernández Perez. The first two
\end{abstract}

\footnotetext{
${ }^{1}$ Doctora en Estudios Transdiciplinarios de la Cultura y la Comunicación. Profesora investigadora en la División Académica de Educación y Artes de la Universidad Juárez Autónoma de Tabasco.

${ }^{2}$ Doctor en Derecho. Profesor Investigador de la División Académica de Ciencias Sociales y Humanidades de la Universidad Juárez Autónoma de Tabasco.
} 


\section{Periódico do Núcleo de Estudos e Pesquisas sobre Gênero e Direito Centro de Ciências Jurídicas - Universidade Federal da Paraíba V. 7 - No 04 - Ano 2018 - Spanish Edition ISSN | 2179-7137 | http://periodicos.ufpb.br/ojs2/index.php/ged/index}

are relatives, they are cousins, the last one is only part of the same community. The analysis is based on the Theory of Social Representations, defined in social practices and social representations by Jean Claude Abric (2001) "[...] as a functional vision of the world that allows the individual or group to give meaning to their behavior and to understand the reality through their own systems of references and to adapt and to define a place for them in this way". According to this idea, the social representations have a structure, which is distinguish by its elements: the central core and the peripheries. The first one has a generate and organizing function, and the other one has a function of concretion, regulation and defense. With these two elements and their components, these indigenous women explain what family representation means for them.

Key words: Social representation, families, women, immigrant, indigenous people

\section{Introducción}

El motivo de este análisis es retomar la Teoría de las Representaciones Sociales contenida en el libro Prácticas Sociales y

Representaciones de Jean-Claude Abric (2001), y emprender su aplicación al caso de las mujeres indígenas migrantes de Tecoluta $1^{\text {a }}$ del municipio de Nacajuca, Tabasco. México. Lo anterior obedece a dos razones: La primera tiene que ver con la teoría, puesto que se considera una herramienta útil para entender la manifestación simbólica de las relaciones establecidas en la experiencia humana. La segunda porque las entrevistas realizadas ofrecen filones muy ricos, que permiten su exploración de manera beneficiosa. El presente escrito está elaborado de tal forma que busca entretejer la teoría con las prácticas sociales expresadas por las mujeres de nuestro caso de estudio.

\section{Los Pueblos Indígenas:}

Sin embargo, no se puede dejar de lado que se trata de una comunidad considerada como indígena. En este sentido, la Constitución Política de los Estados Unidos Mexicanos (Diputados, 2018), en su artículo Segundo reconoce que México tiene una composición pluricultural sustentada originalmente en sus pueblos indígenas que son aquellos que descienden de poblaciones que 


\section{Periódico do Núcleo de Estudos e Pesquisas sobre Gênero e Direito Centro de Ciências Jurídicas - Universidade Federal da Paraíba V. 7 - No 04 - Ano 2018 - Spanish Edition ISSN | 2179-7137 | http://periodicos.ufpb.br/ojs2/index.php/ged/index}

habitaban en el territorio actual del país al iniciarse la colonización y que conservan sus propias instituciones sociales, económicas, culturales y políticas, o parte de ellas.

La Organización Internacional del Trabajo, en el Convenio 169 indicó que los pueblos indígenas son descendientes de poblaciones que habitaban un país o una región geográfica determinada durante la conquista o la colonización o el establecimiento de las actuales fronteras estatales y conservan sus propias instituciones sociales, económicas, culturales y las instituciones políticas (Burger, 2014).

En México existen por lo menos 57 grupos étnicos reconocidos y su población aumentó de dos millones en 1895 a seis millones en el 2000 (Heredia, 2006). Sin embargo, su representación con respecto al demás resto de la población ha disminuido de $16.1 \%$ en 1895 a 7.1 en el 2000. La Comisión Nacional para el Desarrollo de los Pueblos Indígenas (CDI) y la Comisión Nacional de la Población (CONAPO) señalan que existen 62 pueblos originarios, que habitan en 24 Estados de la República Mexicana y se asientan principalmente en el sudeste mexicano y en menor grado en el centro. En cinco de estos Estados el número de indígenas alcanza más del millón de habitantes. La lista es liderada por Oaxaca, Chiapas, Veracruz, Puebla y Yucatán.

De acuerdo con la Comisión Nacional para el Desarrollo de los Pueblos Indígenas (CDI), en Tabasco existen 78,759 indígenas de origen chontal. Los hombres y mujeres indígenas se agrupan de acuerdo a las comunidades o poblaciones en donde conviven y trabajan junto con sus vecinos. Hablan el mismo idioma, celebran las mismas fiestas, mantienen sus ideas y costumbres. A lo largo de los años, estos pueblos indígenas han aprendido a vivir en los ecosistemas más variados del territorio mexicano (Navarrete, Pueblos indígenas de México, 2010).

Desafortunadamente algunos conceptos negativos se asocian con el término indígena, como es el de "ser pobre" que corresponde en gran medida a la realidad de estos pueblos, pues padecen de un alto grado de marginación no solamente de las autoridades sino de los propios ciudadanos que consideran que al ser "mayoría" tienen mayores derechos que esta minoría. Asimismo, esta separación no sólo se da en el plano 
político sino social y económico que es el resultado de siglos de explotación y discriminación que se ha hecho más visible en los últimos años, afectando en gran medida su desarrollo y dificultando su florecimiento. Esto también justifica el supuesto atraso de su cultura y forma de vida, sin considerarse que en realidad son comunidades tradicionalistas, pero no son contrarias al progreso y la modernidad ya que, de hacerse de esta forma, se niega o menosprecia las formas de racismo, explotación y discriminación que son parte de la marginación de la que son objeto (Navarrete, 2008).

Generalmente se tiene a considerar que estos pueblos no tienen derechos. Y aunque la propia Carta Magna reconoce y garantiza su libre determinación y autonomía para decidir sus formas de convivencia y organización social, económica, política y cultural, también tienen derecho a la alimentación, a un medio ambiente sano, a una vivienda digna, a la educación, el esparcimiento, la práctica del deporte y a los bienes y servicios que preste el Estado (SEGOB, 2014).

En virtud de ser considerados como una minoría, tienen derecho a profesar su propia cultura, a profesar o practicar su propia religión, a utilizar su propio idioma, en privado o en público, así como a participar en la vida cultural, religiosa, social, económica y pública. Esto puede ser paradójico porque, por un lado, la ley no hace distinción para su respeto y cumplimiento; sin embargo, su libre determinación incluye su propia organización política y la posibilidad de decidir sobre su desarrollo económico, social y cultural. Tienen derecho a la autonomía y al autogobierno sobre asuntos internos y locales y a vivir en libertad, paz y seguridad (SEGOB, 2014).

En ese mismo tenor de ideas, el Pacto Internacional de Derechos Civiles y Políticos establece en su artículo 27 que los Estados en los que existan minorías étnicas, no se les negará a sus miembros el derecho a tener su propia vida cultural, a profesar su propia religión y a emplear su propio idioma. La Declaración sobre los Derechos de las Personas pertenecientes a Minorías Nacionales o Étnicas, Religiosas y Lingüísticas establece la obligación para los Estados de proteger la existencia y la identidad nacional o étnica, cultural, religiosa y lingüística dentro de sus territorios, además de fomentar las condiciones para la promoción de esa identidad (CNDH, 2012). 
Desafortunadamente, en estos grupos existe una marcada división del trabajo de acuerdo al sexo, lo que otorga una visión distinta de las tareas que cada género realiza. Las mujeres son las encargadas del trabajo doméstico y el cuidado y crianza de los hijos, mientras que los varones son los responsables de las actividades agrícolas. Sin embargo, las mujeres además de realizar sus obligaciones, deben hacer $\mathrm{y}$ vender artesanías, emplearse como jornaleras, entre otras. Desde muy temprana edad, las mujeres van aprendiendo a realizar labores específicas dentro del ámbito doméstico y se olvidan del ámbito educativo que se prioriza para la educación masculina, por lo que son obligadas a abandonar la escuela para colaborar con las tareas domésticas (Olivera, 2001).

Las mujeres suelen vivir una vida distinta a la que experimentan las mujeres de comunidades urbanas. Los roles de género son más marcados en las comunidades indígenas $\mathrm{y}$ es difícil romper con los esquemas establecidos por el machismo y las costumbres patriarcales. Principalmente porque los roles sexuales no privilegian la repartición de tareas, ni asegura que estas serán equitativas; sobre todo porque lo masculino prevalece sobre lo femenino. Lo público es accesible para el hombre, mientras que el ámbito privado tiene que ver con el hogar y se destina a la mujer (Araiza, 2006).

Cada pueblo indígena en México tiene su propia forma de organización política, aunque se encuentran bajo la influencia de lo que marca la norma Constitucional. Cada comunidad indígena elige a las autoridades que los gobiernan, siempre respetando las reglas y doctrinas que han sido parte de su tradición. La comunidad indígena tiene a su favor la riqueza pluricultural, producto del mestizaje que los une, sin que importe la escases económica y las condiciones en la que muchos viven. Aceptan la situación que en la que se encuentran y la viven de manera digna pues saben que son ricos en el conocimiento de sus tradiciones místicas, el colorido de sus atuendos que ellos mismos elaboran y venden para su sobrevivencia; $\sin$ soslayar la gastronomía propia de ellos (Navarrete, 2008).

El idioma es otro elemento importante, pues hacen esfuerzos por conservarlo, sin importarles ser sujetos de discriminación por la burla de los demás que no comprenden la riqueza 
cultural que esto representa. A diferencia de los citadinos, los indígenas abren las puestas de su pueblo y cobijan a sus hermanos; cuidan y aman la naturaleza y la protegen y veneran, pues saben que de ella han hecho su medio de subsistencia y ella les provee de todo lo necesario para vivir de manera digna (Mayo, 2015).

\section{Los Pueblos Indígenas En Tabasco,} México

Tabasco es un Estado que se compone de 17 municipios y se localiza en el Sureste de la República Mexicana. Cuando en el siglo XIX se rompió con el orden colonial, Tabasco se convirtió en un incipiente estado más de la naciente República Mexicana. En 10 de los 17 municipios se hablaba el chontal, y representaban el 10 por ciento de la población total; y durante el siglo $\mathrm{XX}$, las comunidades chontales se transformaron y experimentaron cambios fundamentales en su organización social: en su estructura familiar, en sus formas de producción, en su relación con el medio ambiente, en su forma de regir la vida política; además de cambios en el vestido, el uso del idioma $\mathrm{y}$ en sus creencias religiosas (Flores, 2006).

El término chontal es un vocablo mexicano o náhuatl que significa "extranjero", origen con el que se identifica este grupo con los chontales de Oaxaca, aunque los tabasqueños prefieren denominarse "yokot'an" que se traduce como "el pueblo que habla yoko ochoco", o "el que habla la lengua verdadera" (Flores, 2006).

De acuerdo con el Censo General de Población y Vivienda de 2010, elaborado por el Instituto Nacional de Estadística y Geografía (INEGI), existen 60 mil 526 personas de 5 años y más que hablan alguna lengua indígena, lo que representa el 3\% de la población total en la entidad. La Comisión para el Desarrollo de los Pueblos Indígenas (CDI) aseguró que existen 120 mil indígenas, debido a que reconoce a los nativos que tienen antecedentes ancestrales, aunque no hablen alguna lengua. Y los municipios que concentran más de cinco mil habitantes indígenas son: Centla, Centro, Macuspana, Nacajuca, Tacotalpa y Tenosique. La mayor parte de estas comunidades cuentan con aproximadamente dos mil quinientos habitantes, los cuales viven en situación de pobreza y atraso, de acuerdo 
a los datos proporcionados por el Fondo Monetario Internacional y el Banco Mundial (Copladet, 2015).

La década de los cincuenta en Tabasco, se caracterizó por una fuerte agresión cultural hacia los pueblos indígenas pues se prohibió el uso de su lengua y el vestido indígena; a tal grado que se negó la existencia de este grupo minoritario en el Estado. El boom petrolero produjo la contratación temporal de aquellos indígenas que tuvieran la edad para trabajar, pero sólo obtuvieron contratos temporales y con baja remuneración debido a la falta de habilidades técnicas, capacitación y adiestramiento oportuno; por lo que los beneficios se presentaron en menor grado para ellos, rezagándolos en aspectos como alimentación, salud, educación, trabajo y vivienda, afectando sus intereses personales, colectivos $\mathrm{y}$ comunitarios.

En 1990, la población indígena de entre 0 a 14 años era de $43.9 \%$ pero para el año 2010, disminuyó a 29.6\%, con lo que se demuestra que, en la actualidad, las mujeres indígenas tienden a disminuir el número de hijos, debido a un mayor nivel educativo y acceso a servicios de salud reproductiva que propician mejores condiciones de vida.
Las mujeres de 15 a 49 años registraron un promedio de 2.7 hijos que se redujo a 1.7 en el resto de la población en ese rango de edad. De igual forma, el analfabetismo tiende a bajar; sin embargo, las mujeres han resentido en mayor medida este rubro que los varones, toda vez que éstos últimos se encuentran en mejor posición, principalmente (Copladet, 2015).

Sin embargo, en cuanto al empoderamiento femenino, en las comunidades indígenas aún se está lejos de alcanzar su equilibrio. $\mathrm{La}$ Constitución Mexicana (Diputados, 2018) establece en su artículo 4 que tanto el hombre como la mujer son iguales ante la ley, pero en las comunidades indígenas tienen su propia forma de organización en este aspecto. El reclamo de los pueblos indígenas es que el Estado les reconozca su existencia, así como su derecho a la autonomía; sin embargo, los patrones culturales y ancestrales en los que se han desarrollado imposibilitan, por ejemplo, la igualdad de género, pues los papeles, como se ha señalado, están delimitados y están ya establecidos, por lo que, ante la globalización imperante, refuerzan sus lazos comunitarios y sociales y buscan mantener su status quo. 
Es importante puntualizar que el empoderamiento se manifiesta mediante la distribución del poder. En lo que respecta a las mujeres, la ideología patriarcal es el mayor desafío ya que se requiere transformar las estructuras e instituciones que refuerzan la discriminación de género. Este empoderamiento se debe realizar en tres dimensiones: la personal, la colectiva y la de las relaciones cercana. La primera tiene que ver con la forma en como la mujer se visualiza como persona, la segunda tiene relación con la organización a la que pertenece; y la tercera, a las relaciones que se establece con su familia (Pérez M. , 2008).

Nacajuca es un municipio integrado por 82 poblados, rancherías y ejidos. Para el año 2000 su población oscilaba sobre los 80,772 habitantes. Sus principales comunidades indígenas son: Tecoluta, Tapotzingo, San Simón, Guaytalpa, Guatacalca, Tucta, Oxiacaque, Olcuatitlan, San Isidro, entre otros. En el caso del poblado Tecoluta, 1ra sección, perteneciente al municipio de Nacajuca, que colinda con el municipio de Centro y su capital Villahermosa, en el año 2000 contaba con una población de 1,013 personas, de los cuales 870 hablaban chontal (Pérez J. , 2007).

El tipo de familia es nuclear y extensa, y se constituye por el padre, la madre y los hijos solteros. La familia extensa incluye a uno o dos hijos casados con su esposa e hijos; por lo que son neolocales, si viven en casas independientes de los padres o viven en una misma casa los hijos casados con los padres; en este caso se considera patrilocal. Por lo general, el padre proporciona a sus hijos una parte del terreno de la casa (traspatio, solar o terreno) para que construya su propio hogar. Así la hija se separa de su familia, para vivir en la casa de su esposo o de manera independiente de la casa de ellos (Pérez J. , 2007).

\section{Algunas Visiones De Familia En Tecoluta $1^{\mathrm{a}}$. Nacajuca.}

En la comunidad indígena de Tecoluta $1^{\mathrm{a}}$ en Nacajuca Tabasco, algunas mujeres están decidiendo migrar, debido a la necesidad económica que viven sus familiares. Su proceso migratorio es temporal, lo hacen de manera semanal, de lunes a viernes salen de su lugar de origen a Villahermosa, capital del Estado de Tabasco, para 
regresar a su hogar en Tecoluta $1^{\mathrm{a}}$ cada fin de semana.

Es importante destacar que anteriormente las personas del campo y las comunidades indígenas migraban a las ciudades porque las veían como una forma de salir de su situación de pobreza y mejorar sus condiciones de vida. De igual forma algunas personas llevaban a cabo una migración temporal, es decir, combinaban el trabajo asalariado con las labores del campo siendo la migración mayoritariamente masculina. A partir de la década de los 90 se apreció un incremento en el volumen de migración indígena que incluía ya no sólo al varón, sino que abarcaba a las mujeres y a los niños. En cuanto a la migración de estos dos grupos, en los últimos años predominan las mujeres en el total de migrantes interestatales del país y cuya edad oscila entre los 15 y los 29 años, por lo que a veces se desplazan con los hijos. Ahora, se considera que las mujeres le dan un nuevo rostro al fenómeno migratorio, siendo en muchos casos madres solteras, divorciadas, jefas de familia, indígenas, etc. (Cárdenas, 2014)

El patrón social que por décadas ha estado marcado en las familias, de que el hombre es el que debe proveer y mantener tanto a la esposa como a los hijos, lo están rompiendo estas mujeres y lo hacen en dos sentidos, el primero al decidir migrar y dejar el hogar de manera temporal; y, el segundo, al ir en busca de mejoras económicas que permitan a ellas y a su familia, una mejor calidad de vida. Veamos pues qué sucede con ellas y su familia durante este proceso migratorio y cuáles son las representaciones sociales que se manifiestan.

\section{○ Lo nuclear}

Abric (2001) indica que "el núcleo central es el elemento esencial de toda representación social constituida y que puede de algún modo ir más allá del simple marco de objeto de la representación para encontrar directamente su origen en valores que lo superan, y que no necesitan aspectos figurativos, esquematización, ni concreción."

Las representaciones sociales están constituidas alrededor de un núcleo central que determina la significación y dispone la cohesión de sus componentes. Sin ellos no se instauraría ningún núcleo, por tanto, la representación sería imposible. Para edificarse, el núcleo central cuenta con dos funciones:

La generadora: que es el elemento mediante la cual se crea y se transforma la significación de los otros 
elementos constitutivos de la representación. Es por su conducto que esos elementos toman un sentido, es decir, un valor.

La organizadora: que constituye el núcleo central que determina la naturaleza de los lazos que unen, entre ellos, los elementos de la representación. Es, este sentido, el elemento unificador y estabilizador de la representación.

En cuanto a la representación social de la familia en el estudio de caso, se observa como núcleo central al conjunto conformado por madre, padre y hermanos; por lo que la configuración de la familia no varía ni se aparta de los esquemas tradicionales sino al contrario: constituye un estereotipo que no se ha desligado de la realidad social. En él se configura una jerarquía en el orden de lo económico y político, la cual gira en torno a la madre. Este actor social norma las reglas de la administración familiar y permite la interacción del grupo, al tiempo que se constituye como un símbolo de poder. Ella, la madre, soporta la significación Familia.

A pregunta expresa acerca del destino del dinero que ganan por su trabajo, las mujeres, objeto de las entrevistas, contestaron:
"Doy a mamá los gastos de la casa. "Cuando llego a la casa se lo doy a mi mamá y ella me da para ahorro o gastos míos".

"Se lo doy a mamá una parte y la otra es para pagar estudios que apenas tres meses empecé" (Estrada, 2016).

Estas prácticas culturales pueden ser explicadas desde la función organizadora.

En la misma dimensión de centralidad, las hijas manifiestan una relativa autonomía, debido a que al salir de su esfera familiar se desplazan por sí mismas, lo que posibilita reproducirla en casa.

No obstante, el control lo sigue ostentando la madre, porque hay una sujeción espacial afectiva permitida por la comunicación telefónica cotidiana que mantienen. De este modo se ejecuta una función generadora, puesto que la movilidad espacial reordenó la representación de familia tradicional, resignificándola. 
Al cuestionarles sobre qué es lo que más extrañan de su comunidad ahora que trabajan fuera de ella, contestaron:

"A mi mamá, pero tengo que trabajar para ayudarla en la casa".

“A mi mamá, pero como tengo que trabajar, pero poco a poco ya se me quitó, me gusta acá también".

"Mi mamá, mi familia, antes pero ya me acostumbré y además me gusta venir acá a trabajar" (Estrada, 2016).

A partir de este discurso se visiona una familia tradicional indígena con una condición económica precaria, que modifica su noción de sí misma al responder a este agente agresor.

\section{○ Lo periférico}

En lo que refiere a este aspecto, Abric (2001) señala que: "Los elementos periféricos se organizan alrededor de un núcleo central [...] constituyen la interface entre el núcleo central y la situación concreta". Más adelante, el autor precisa que los elementos periféricos cumplen con tres funciones en el proceso de la representación social:

1.Función concreción: resulta del anclaje de la representación de la realidad y permiten revestirla en términos concretos, comprensibles $\mathrm{y}$ transmisibles de inmediato. Integran los elementos de la situación en la que la representación se produce, hablan del presente y de lo vivido del sujeto. La noción de familia en este caso de estudio queda manifiesta en la primacía que adquieren las festividades, ya que a través de ellas se concretiza el concepto de familia tradicional, basado en valores religiosos. De este modo, las fiestas que fortalecen la integración de grupo son la del santo patrono San Pedro y Pablo Apóstol y la de día de las Madres, fechas trascendentales para ellas, porque cohesionan los valores y afectos familiares.

"Nada más cuando es la fiesta del santo patrono del pueblo y el día de las madres"

"San Pedro y Pablo Apóstol el patrono de la parroquia y el día de las madres" 
"Las madres y el patrono del

pueblo, San Pedro y Pablo

apóstol' (Estrada, 2016).

2. La función regulación es más

flexible que los elementos centrales que desempeñan un papel esencial en la adaptación de la representación a las evoluciones del contexto [...] frente a la estabilidad del núcleo central constituyen el aspecto móvil y evolutivo de la representación.

Aun cuando la familia se caracteriza por su rasgo tradicional, en ella se permite la movilidad de los actores. Al aparecer una enfermedad se inicia un proceso de movilidad espacial, donde el sujeto transita por un tiempo determinado, generándose una rotación de apego y desapego. El ir y venir regula la afectividad y ésta fortalece el lazo de unión de la familia.

"Al principio triste, pero está bien, yo regreso y está todo bien, ya no me siento así, ya me acostumbré."

"Pues al principio mal porque dejé a mi familia, pero poco a poco me acostumbré, ahora ya me acostumbré y sé que voy a llegar a mi casa. [...]

Tengo que venir trabajar por que no voy a depender de mis papás, yo ya tengo mayor de edad, además para cuando se necesite dinero aquí en la casa, medicina, comida, lo que sea."

"Tengo que ir a trabajar, ¿de dónde saco dinero?, el dinero no alcanza para otras cosas, para doctor, para comida y cosas de la casa, para mis papás. [...] No mal, bien, porque regreso a ver a mi mamá y a todos. Para ayudar en la casa, a mi mamá y a mi familia." (Estrada, 2016).

El núcleo central de una representación ya lo dijimos- resiste al cambio, puesto que su transformación ocasionaría un trastorno completo. Por tanto, el sistema periférico funciona como el sistema de defensa de la representación Constituye lo que Flament llama su «parachoques» (Abric, 2001).

En el caso que se aborda, los chismes afectan al núcleo $\mathrm{y}$ en 
consecuencia a su representación de familia. El escarnio social originado por la transformación de la representación familiar de la comunidad ofende a la familia de este estudio de caso y se constituye como una amenaza.

Ante esta situación, irrumpe la función de defensa o parachoques, representada en la confianza y el diálogo entre los miembros de la familia. De la misma manera se fortalece el honor familiar a partir de la violencia física simbolizada.

Con todas las vicisitudes enfrentadas, la madre se mantiene como el eje fundamental en esta representación; es la que simboliza el poder.

"La gente de la comunidad, de mi comunidad, es muy chismosa, dicen chismes de uno y no me gusta, ya dicen que me casé y no es cierto. Mi papá dice que si escucha a alguien que dice eso le saca el machete. No gusta a mi papá eso, ya lo platicamos en la casa y no gusta a nadie. Son chismosos allá"
"Me siento bien aquí en mi casa, me gusta venir a trabajar, porque allá es muy mentirosa la gente, mucho chisme, mucho chisme, ya dijeron que las mujeres salen por esposo o embarazo y eso no le gusta a mi familia, pero yo llego todos los fines de semana $y$ sabemos que no es cierto, dicen que me casé y no es cierto. Mi papá se enoja, mi mamá dice que no importa que no haga caso, pero mi papá dice que lo va a matar si lo escucha de alguien. Se enoja, pero no nos importa a nosotros, siempre estamos juntos y platicamos de todo." "Me gusta a veces no venir aquí a la comunidad, sólo por mis papás sí los extraño, pero las personas hablan mucho de las mujeres que salen a trabajar, dicen que salen porque se fueron con el marido que 
las embarazó. No me gusta eso, pero no nos importa, ahora nadie lo dice en mi casa, sólo en la calle y mi hermana se lo conto una amiga de ella, pero mi papá dice que si lo escucha apalea al que lo diga. La gente es mucho chisme."

(Estrada, 2016)

\section{Reflexiones Finales}

Después del recorrido realizado, interpretamos que la representación social de familia configurada en nuestro estudio de caso, parte de una concepción tradicional, pero que al surgir una incidencia tiene que flexibilizarse. La salud de la madre obliga al núcleo a resignificarse para subsistir. Además, es la madre, quien, a pesar de su estado limitado, permite la regulación; es decir, hay una circunstancia nueva- la salud de la madre- que rige el tránsito y la movilidad como algo permisible. Por este motivo, el objetivo de vida de las mujeres se transforma, su proyecto de vida se sostiene, pero ya no está centralizado en el matrimonio sino en su trabajo, situación acorde con las nuevas circunstancias contextuales.

Con todas las vicisitudes enfrentadas, la madre se mantiene como el eje fundamental en esta representación, es la que simboliza el poder. Ella es quien norma, ella es organizadora porque administra y regula la interacción. Tales operaciones la colocan en una actuación política. En cuanto al padre, al no poder ejercer su función de proveedor, valor esencial en una familia tradicional, está en el mismo nivel del incidente de la salud; esto es, se convierte en un evento adverso a la estabilidad de la representación de familia.

Ambos factores, la enfermedad de la madre y la no provisión del padre, inducen la emigración de las hijas. Pero él también es un vigilante simbólico al constituirse como protector de la familia, concretizado en el ejercicio de la violencia simbólica. Es cuando, entonces, asume la función de defensa ante los discursos externos que amenazan al honor familiar, como corresponde en la familia tradicional. En este último punto, las hijas constituyen una amenaza a la representación de familia tradicional indígena, porque sustentadas en su nueva mirada del 
mundo, permitida por la movilidad, su objetivo de vida se modificó, lo que a su vez transformó la representación familiar inicial.

Este acto, debido al proceso de migración de la comunidad, propiciará cambios en la tradición indígena de Tecoluta, básicamente porque la defensa de su representación se vuelve cada vez más débil ante las necesidades exigidas por el contexto, y el éxito de las jóvenes estudiadas se ofrece como una alternativa cada vez más viable.

Como se puede entender, una nueva configuración de una comunidad implica también la transformación de sus actores, lo que es inherente a una transformación del mundo. Estamos ante una representación de familia que puede ir en pos de otra representación.

\section{Bibliografía}

Abric, J.-C. (2001). Prácticas Sociales y representaciones. . México-Ambassade de France: Coyoacán.

Araiza, A. (2006). Las mujeres indígenas en México: un análisis desde la perspectiva de género. Quaderns-e de l'institut Catalá d'Antropología, 1-17.
Burger, J. (2014). La protección de los pueblos indígenas en el sistema internacional. En J. F. Beltrao, Derechos Humanos de los grupos vulnerables. Manual (págs. 213-239). Barcelona: Red de Derechos Humanos y Educación Superior.

Cárdenas, P. (2014). Migración interna e indígena en México: enfoques y perspectivas. Instersticios Sociales No. 7, 1-28.

CNDH. (2012). Derechos Humanos de los Pueblos Indígenas en México. México: Comisión Nacional de los Derechos Humanos.

Copladet. (2015). Programa Especial para la Atención de los Pueblos Indígenas 2013-2018. Tabasco: Secretaría de Desarrollo Social.

Diputados. (27 de 08 de 2018). Constitución Política de los Estados Unidos Mexicanos. Obtenido de Cámara de

Diputados: http://www.diputados.gob.mx/LeyesBib lio/pdf/1_270818.pdf 
Estrada, C. (2016). Las representaciones sociales en las mujeres indígenas de Tabasco, sobre su proceso de migración. México: UJAT.

Flores, J. (2006). Chontales de Tabasco. México: CDI.

Heredia, E. (2006). Los pueblos indígenas en México y la CNDH. Casa del Tiempo, 53-61.

Mayo, K. (2015). Las niñas indigenas en México y su derecho a la educación. Genero e direito, 16-33.

Navarrete, F. (2008). Los pueblos indígenas de México. Pueblos indigenas del México contemporáneo. México: CDI.

Navarrete, F. (2010). Pueblos indígenas de México. México: MacMillan.

Olivera, M. (2001). Mujeres indígenas en México. México: Mujeres indígenas, seminario de GIMTRAP.

Pérez, J. (2007). Desarrollo local en el trópico mexicano. Los camellones chontales de Tucta, Tabasco. México:

Universodad Iberoamericana.

Pérez, M. (2008). Empoderamiento de las mujeres indígenas de Tabasco. El papel de los fondos regionales de la CDI. Cuicuilco Vol. 15, No. 42, 165-179.

SEGOB. (2014). Pueblos indígenas. México: Secretaría de GobernaciónUnidad para la Promoción y Defensa de los Derechos Humanos. 